\title{
Dynamics of charge trapping by electron-irradiated alumina
}

\author{
C. Bonnelle* and P. Jonnard \\ Laboratoire de Chimie Physique-Matière et Rayonnement, UPMC University of Paris 06, CNRS-UMR 7614, \\ 11 rue Pierre et Marie Curie, F-75231 Paris Cedex 05, France
}

(Received 23 March 2010; revised manuscript received 12 July 2010; published 24 August 2010)

\begin{abstract}
Electron trapping in the oxygen vacancies of various alumina samples is analyzed using $\mathrm{x}$-ray and optical emissions induced by electrons. Observed x-ray intensity variations show the presence of charges in the sample. Information on the ionicity is deduced from the electron distribution around aluminum, observed by $\mathrm{x}$-ray emission between 50 and $300 \mathrm{~K}$. It is shown that the stable defects are oxygen vacancies with one trapped electron. Electron trapping in these defects is analyzed by cathodoluminescence as a function of the structural characters of the sample, of the temperature, and of the irradiation conditions. Conditions for weak trapping can be deduced from these experiments.
\end{abstract}

DOI: 10.1103/PhysRevB.82.075132

PACS number(s): 72.20.Jv, 78.70.En, 78.60.Hk

\section{INTRODUCTION}

In a real crystal defects are impurities and lattice defects, such as vacancies, interstitial atoms, and dislocations. Each defect is associated with a quantum state involving a ground level and one or more excited levels and these levels are localized in the gap band of a nonconductor solid. The defects, which cause a deficit of negative charge, have a large probability to capture an electron, leading to a partial return to electroneutrality.

In oxides, the most common defects are the oxygen vacancies, which are electron traps. An oxygen vacancy having trapped one or two electrons becomes an $\mathrm{F}^{+}$or an $\mathrm{F}$ center. Impurities can also be present; only the impurities with oxidation degree higher than the one of the metallic element are electron traps.

In an insulator irradiated by electrons with incident energy $E_{0}$ of $1 \mathrm{keV}$ or more, numerous electron processes are possible: slowing down and thermalization of incident electrons, impact ionization of the core and valence electrons, and simultaneous creation of holes in atomic levels and in the valence band, impact excitation to unoccupied defect levels present in the band gap, creation of core or valence excitons, and electron recombination from occupied defect levels to the atomic and valence holes.

Charge effects due to trapping of thermalized electrons in highly excited defect levels are also present, accompanied by the reverse process, or thermal detrapping, and by the radiative and nonradiative de-excitation of electrons in the highly excited defect levels to their ground level. Direct electron trapping from the conduction band to a deep defect level need not to be considered since its probability is negligible. In the same way, the creation of defects under electron irradiation does not occur in the considered energy domain.

In the oxides, it was generally assumed that the ionic charges are the integer values that correspond to the conventional chemical assignment of the oxidation state. ${ }^{1}$ In this model, the bond should have an ionic character. Indeed it has been shown for $\mathrm{MgO}$ that the charge on oxygen is on the order of unity. ${ }^{2}$ Consequently the most stable defect is the $\mathrm{F}^{+}$ center (oxygen vacancy having trapped an electron). An $\mathrm{F}^{+}$ center can trap an electron and becomes an F center. As a result, the dynamics of the electron trapping in insulator oxides can be deduced directly from the $\mathrm{F}^{+}-\mathrm{F}$ transformation. ${ }^{3}$

Electron-induced $\mathrm{x}$-ray emission spectroscopy (EXES) and cathodoluminescence can be used to study the charge effects and the trapping-detrapping in insulators. These emissions compete with the nonradiative transitions, such as $\mathrm{Au}-$ ger and multiphonon processes. In this paper, they are observed for alumina at different temperatures and discussed in connection with the model developed in Ref. 4.

Information on the charge effects is deduced from following EXES parameters: (a) the intensity variation in an $\mathrm{X}$-ray characteristic line as a function of the incident-beam current, called charge curve. In the absence of charge effect, the number of photons increases linearly with the electron current. When charge effect is present the increase in number of photons is not linear and less rapid.

(b) The intensity variation in an x-ray characteristic line as a function of the accelerating voltage of the incident electron beam, called excitation curve. In the absence of charge effect and above the threshold, the number of photons increases with the accelerating voltage according to a power law. The presence of a charge induces a retarding potential, which slows down the incident electrons and the number of photons increases more slowly.

(c) The intensity of $\mathrm{x}$-ray emission structures, located toward the higher energies of the valence-band emission and characteristic of the radiative recombination from defect states to a core hole. ${ }^{5}$ The defect states are either initially occupied or filled by excitation of valence or core electrons or by trapping of an incident electron. Filling of a defect state is studied by comparing the intensity of the structure present in the band gap to the intensity of the valence emission band.

At the position of oxygen vacancies, the electron distribution is due to the surrounding cations. In alumina, the electron states, associated with $\mathrm{F}^{+}$and $\mathrm{F}$ centers and localized in the band gap, have the Al $s p$ character and can be observed owing to their $\mathrm{Al} K$ or $\mathrm{Al} L$ emission.

The variation in the number of $l$-symmetry valence electrons around the cation can also be measured comparatively in oxide and metal using EXES. It is possible to deduce from such measurements information on the ionicity. ${ }^{2}$ This is made by analyzing the valence-band emission and an atomic 
line of same initial state, as a function of photon energy at fixed voltage and current, and by then determining the intensity ratio of the two emissions.

By cathodoluminescence, emissions characteristic of each type of defects are observed. These emissions correspond to electron transitions between an excited state and the ground state of the defect. Their shape is strongly temperature dependent. Their broadening is directly related to the electronphonon interactions.

All these parameters have been measured for $\mathrm{Al}_{2} \mathrm{O}_{3}$. Since the radiative transitions depend on the electron distributions, one expects them to vary with a change in the atomic structure and the preparation conditions of the sample. The defect states are strongly coupled to the phonons hence their dependence on the temperature. Moreover, the charge effect depends on the incident electron energy, electron density, and irradiation time. Consequently, measurements have been made for various samples as a function of the crystal arrangement, the temperature, and irradiation conditions.

\section{EXPERIMENT}

An $\alpha-\mathrm{Al}_{2} \mathrm{O}_{3}$ (0001) single crystal of $10^{-3}$ purity was used. The main impurities are $\mathrm{Si}(500 \mathrm{ppm}), \mathrm{Ca}(150 \mathrm{ppm})$, and $\mathrm{Fe}$ (250 ppm). The sample was mechanically polished. The single crystal was annealed in air according to the following conditions: $1 \mathrm{~h}$ at $1000{ }^{\circ} \mathrm{C}$ (sample 1 ); $4 \mathrm{~h}$ at $1500{ }^{\circ} \mathrm{C}$ (sample 2); $4 \mathrm{~h}$ at $1700{ }^{\circ} \mathrm{C}$ (sample 3); and $24 \mathrm{~h}$ at $1700{ }^{\circ} \mathrm{C}$ (sample 4). The temperature was increased at the rate of $100{ }^{\circ} \mathrm{C} / \mathrm{h}$ and decreased at $50{ }^{\circ} \mathrm{C} / \mathrm{h}$. The polycrystalline $\alpha-\mathrm{Al}_{2} \mathrm{O}_{3}$ sample is a powder obtained from JohnsonMatthey. The amorphous sample $\left(\alpha-\mathrm{Al}_{2} \mathrm{O}_{3}\right)$ is a film of 150 nm thickness.

The samples were irradiated at room temperature in a UHV chamber of the IRIS multiple-technique apparatus. ${ }^{6} \mathrm{~A}$ Pierce electron gun, $0-10 \mathrm{kV}, 0-10 \mathrm{~mA}$, produces the electron beam. The beam can be focused to a 5-20 mm diameter. The working pressure is around $10^{-9}$ mbar when the electron gun is on. The samples were irradiated with an electron beam of incident energy varying between 1 and $4 \mathrm{keV}$. The electron current density varied between 0.15 and $4 \mathrm{~mA} / \mathrm{cm}^{2}$. After each annealing, the sample was irradiated for several days during which the cathodoluminescence spectra spectra were recorded for the different irradiation conditions.

The incidence angle of the electron beam on the sample is $20^{\circ}$. The optical cathodoluminescence signal is collected in a direction making $20^{\circ}$ with the normal to the sample. It is transmitted through an optical fiber bundle to a glass viewport of the chamber. The other end of the fiber is connected to the entrance slit of a Czerny-Turner grating spectrometer. The spectral interval between 250 and $850 \mathrm{~nm}$ is analyzed with a $300 \mathrm{~nm}$ band pass by means of a 150 grooves $/ \mathrm{mm}$ grating. The detector (Tracor-Northern) is an intensified linear 512 photodiode array. The wavelength scale is calibrated with the help of a Hg-Ar lamp. Simultaneously x-ray emissions were also observed with the high-resolution curvedcrystal spectrometer of the IRIS apparatus. Since the Al $1 s$ threshold energy $E_{\mathrm{s}}$ is equal to $1.5 \mathrm{keV}$, the lower incident
TABLE I. Penetration depth and emissive thickness obtained with $1-4 \mathrm{keV}$ incident electrons in alumina.

\begin{tabular}{ccc}
\hline \hline $\begin{array}{c}\text { Incident electron } \\
\text { energy } E_{0} \\
(\mathrm{keV})\end{array}$ & $\begin{array}{c}\text { Luminescence } \\
\text { penetration depth } \\
(\mathrm{nm})\end{array}$ & $\begin{array}{c}K \text { emission } \\
\text { emissive thickness } \\
(\mathrm{nm})\end{array}$ \\
\hline 1.0 & 10 & \\
1.9 & 30 & 10 \\
4.0 & 85 & 65 \\
\hline
\end{tabular}

electron energy used in the x-ray experiments was $1.9 \mathrm{keV}$. The analyzed depths, or emissive thicknesses, vary as indicated in Table I. Luminescence can be induced by lowenergy electrons. For the $1-4 \mathrm{keV}$ electrons we used, the corresponding penetration depths are shown in Table I.

The temperature of the sample holder can be varied between 50 and $300 \mathrm{~K}$ using a cryocooler that is the first stage of a cryogenic pump. ${ }^{7}$ The temperature is regulated by using a chromel-alumel thermocouple ( $K$ type) with a precision of $5 \mathrm{~K}$.

\section{RESULTS}

\section{A. X-ray emission spectra}

Charge curves have been obtained for an atomic line $(\mathrm{Al} 2 p \rightarrow 1 s)$ at several temperatures T [Fig. 1(a)]. When the
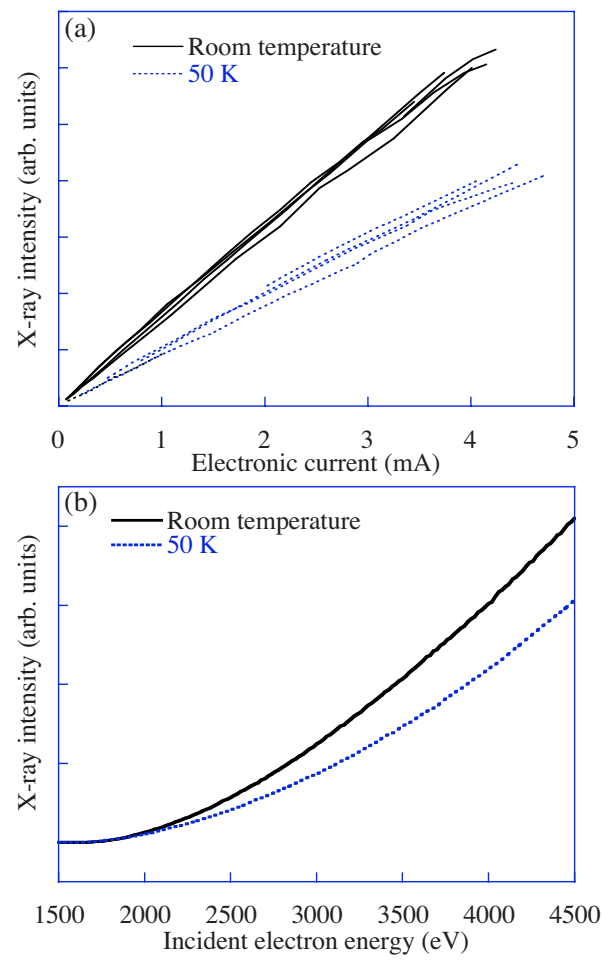

FIG. 1. (Color online) (a) Charge curve at $4 \mathrm{kV}$ and (b) excitation curve at $3 \mathrm{~mA}$ of $\alpha-\mathrm{Al}_{2} \mathrm{O}_{3}$ (sample 2) at two temperatures. The photon energy is taken at the maximum of the $\mathrm{Al} K \alpha$ emission. The various curves in (a) correspond to successive increases and decreases in the electron current. 
charge effect increases, one expects a decreasing of the slope of the curves giving the emitted intensity as a function of the incident electron current. A net increase in the charge with the decrease in the temperature is observed independently of $E_{0}$ and sample characteristics.

The excitation curves show also a decrease in the intensity of x-ray emissions as temperature decreases [Fig. 1(b)]. This is due to the increase in the charge effect at low temperature. In the energy range $E_{0} / E_{\mathrm{s}} \leq 1.15 \quad\left(1560<E_{0}\right.$ $<1800$ ), no decrease in intensity is observed. Charge effect is present in the first atomic layers of the sample at room temperature and no charge increase is detected in this range with our experimental conditions.

At very low temperature, oscillations of the x-ray intensity plotted versus emitted photon energy have been observed for the sample $1 .^{8}$ These oscillations reflect a fluctuation of the electron current used in the production of the $\mathrm{x}$-ray emission. During the irradiation, negative charges flow out through the sample. An accumulation of negative charges repels the incident electrons. Therefore the number of electrons entering the target and creating core ionizations decreases as well as the $\mathrm{x}$-ray intensity. When the charge excess diminishes, the intensity comes back its initial value. Oscillations are observed only for strongly charged samples.

The relative number of $p$-symmetry valence electrons around $\mathrm{Al}$ in metal and oxide is deduced experimentally from the intensity ratio between the $\mathrm{Al} K \beta(3 p \rightarrow 1 s)$ emission band and the $\mathrm{Al} K \alpha(2 p \rightarrow 1 s)$ atomic line of the same initial state. The intensity of an x-ray emission depends on three factors: the number of initial states, here ionized states with an $1 s$ core hole; the density of the final states, $\mathrm{N}(3 p)$ or $\mathrm{N}(2 p)$; and the probability of the $n p \rightarrow 1 s$ transition. Because the initial state of the $K \beta$ and $K \alpha$ lines is the same, the intensity ratio $R=K \beta / K \alpha$ depends only on the final states. The $2 p$ core states are atomic states; their variation with the physicochemical state of the sample and with the temperature is negligible. Then a change in the $K \beta / K \alpha$ intensity ratio denotes a variation in the density of the $3 p$ valence states $\mathrm{N}(3 p)$ and of the $3 p \rightarrow 1 s$ transition probability. From calculations made for $\mathrm{MgO},{ }^{2}$ the prevailing variation is that of the density of the states.

The intensity ratios $R$ have been measured for $\mathrm{Al}_{2} \mathrm{O}_{3}$ and $\mathrm{Al}$ as a function of the temperature and the atomic structure. At room temperature, the results are comparable with those observed for $\mathrm{MgO}^{2}$ One deduces that the charge on oxygen is on the order of the unity.

Change in the $K \beta / K \alpha$ intensity ratio is observed at low temperatures. This change shows that the density of the Al $3 p$ valence states decreases with the temperature. To this decrease corresponds an increase in the ionicity and also of the polarization of the sample. Therefore the charge effect increases inversely to the temperature but this change remains weak.

As already underlined, the electron state associated with an $\mathrm{F}^{+}$center and localized in the band gap has an $\mathrm{Al} 3 s p$ character. From the analysis of the $\mathrm{Al} 3 p$ distribution by EXES, at room temperature, a structure is observed at $\approx 1 \mathrm{eV}$ above the top of the $K$ emission band toward the higher energies, i.e., $\approx 8 \mathrm{eV}$ below the bottom of the conduction band, for the sample 4 of gap width equal to $9 \mathrm{eV}$
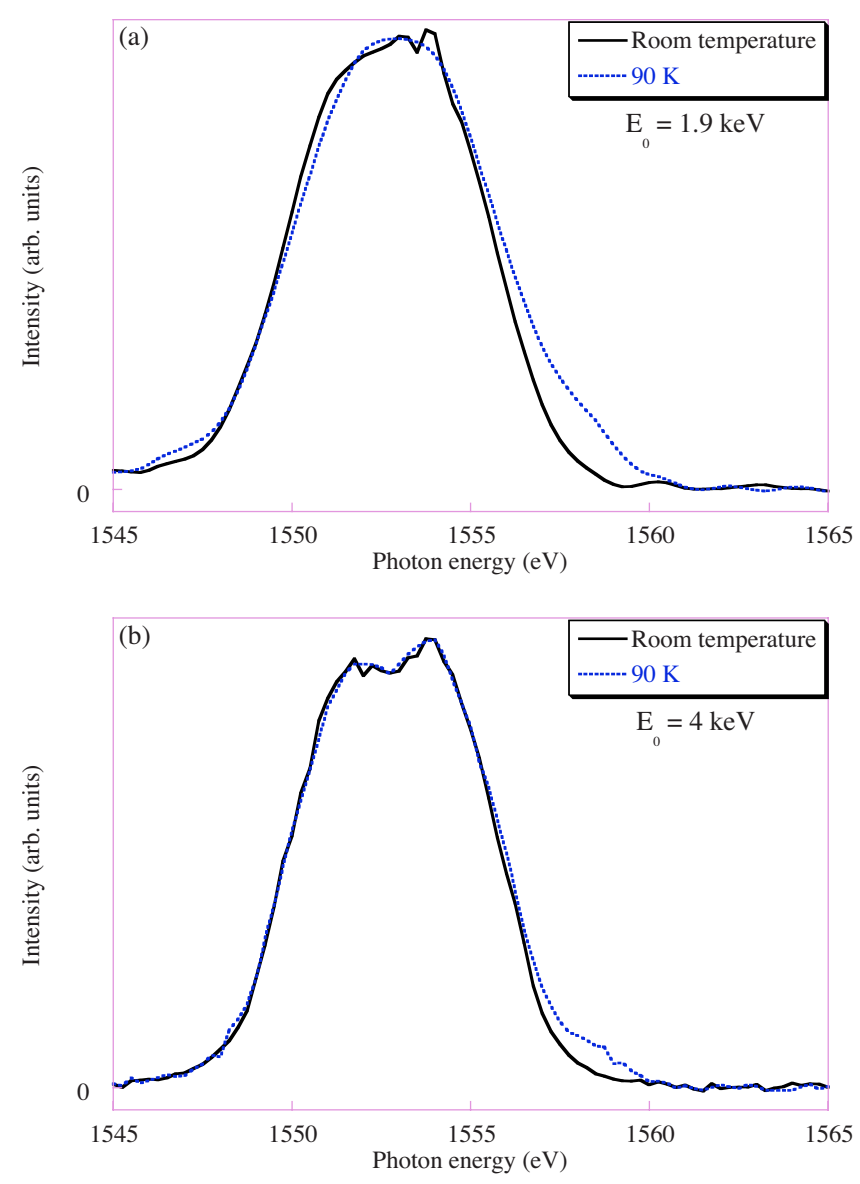

FIG. 2. (Color online) $\mathrm{Al} K \beta$ emission of $\alpha-\mathrm{Al}_{2} \mathrm{O}_{3}$ (sample 4). Spectra at room temperature (solid lines) and $90 \mathrm{~K}$ (dotted lines) with electron current of $1.5 \mathrm{~mA}$ : (a) $1.9 \mathrm{keV}$ and (b) $4 \mathrm{keV}$.

[Figs. 2(a) and 2(b)]. Its intensity increases with both incident electron energy and temperature decreasing. It must be noted that the presence of the inner hole changes the energy of the defect state. By taking into account this effect, we attribute the feature to the transition of one electron from the defect to the $1 s$ core level, leaving a $\mathrm{F}^{+}$center with an electron lacking in its ground level.

Supplementary features are observed from sample 2 between 1 and $3 \mathrm{eV}$ below the bottom of the conduction band; their intensity increases at low incident energy $\left(E_{0} / E_{1 \mathrm{~s}}\right.$ $\approx 1.15$ ) and low temperature but remains always very weak. They can be attributed to the recombination of an electron initially excited from the $1 s$ level to an excited level of an $\mathrm{F}^{+}$ center. Indeed, excitation and recombination probabilities increase at the vicinity of the threshold.

\section{B. Optical emission spectra}

The optical emissions characteristic of $\mathrm{F}^{+}$and $\mathrm{F}$ centers in the first-order reflection (300-550 $\mathrm{nm}$ spectral range) are the only ones considered here. For all samples, at room temperature and incident electron energy $E_{0}$ equal or superior to 1.9 $\mathrm{keV}$, the spectrum is widely dominated by the band at 3.75 $\mathrm{eV}$, characteristic of centers $\mathrm{F}^{+}$of $\alpha-\mathrm{Al}_{2} \mathrm{O}_{3}$. A weak band is observed at $2.9 \mathrm{eV}$; it is known as being characteristic of the 

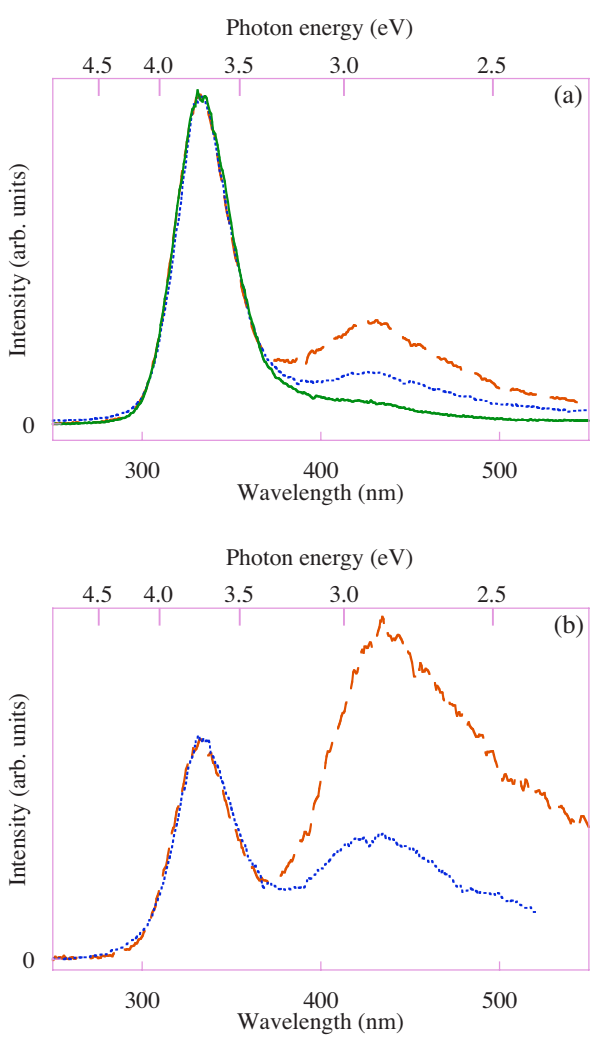

FIG. 3. (Color online) Cathodoluminescence of $\alpha-\mathrm{Al}_{2} \mathrm{O}_{3}$ (sample 2) spectra at beginning of irradiation (dotted line); after 21 $\mathrm{h}$ of irradiation (dashed line): (a) $1.9 \mathrm{keV}$ and (b) $1 \mathrm{keV}$. The solid line in (a) is obtained at $4 \mathrm{keV}$ after $21 \mathrm{~h}$ of irradiation.

emission from F centers [Fig. 3(a)]. Observations are made in the beginning of the electron irradiation and after irradiation of about $20 \mathrm{~h}$ in order to analyze the luminescence at the beginning and the end of the x-ray spectrum. For the sample 2 at $4 \mathrm{keV}$, the $\mathrm{F}$ emission is not detected and no change is observed as a function of the irradiation time $t$ while at 1.9 $\mathrm{keV}$, the intensity of the $\mathrm{F}$ emission increases with $t$ but remains always weaker than the $\mathrm{F}^{+}$emission.

A spectacular change in the optical emission is observed at $1 \mathrm{keV}$ from sample 2 [Fig. 3(b)]. After a short irradiation, the $\mathrm{F}^{+} / \mathrm{F}$ ratio remains bigger than 1 but after a long irradiation, the $\mathrm{F}$ emission becomes the strongest feature of the spectrum; it is a wide band highly asymmetric toward the lower energies. This is not observed for samples 3 and 4 (Fig. 4). A supplementary structure is seen on the spectrum of sample 4; it is situated toward the higher energies of $\mathrm{F}^{+}$ emission at about $4.3 \mathrm{eV}$.

Experiments as a function of the irradiation time are shown in Fig. 5 for sample 3 irradiated under $1 \mathrm{keV}$. At $t$ $=0$, the $\mathrm{F}^{+}$and $\mathrm{F}$ emissions are not resolved from the background. Their intensities increase continuously under irradiation up to about $25 \mathrm{~h}$ then decrease weakly. Beyond $20 \mathrm{~h}$ of irradiation, their shape remains unchanged.

Changes with the temperature are presented in Fig. 6 for sample 2. At low temperature, the $\mathrm{F}^{+}$emission is shifted to the higher energies by $+0.07 \mathrm{eV}$ and its width decreases from 0.41 to $0.33 \mathrm{eV}$. The square of the full width at half maximum as a function of the temperature is plotted in

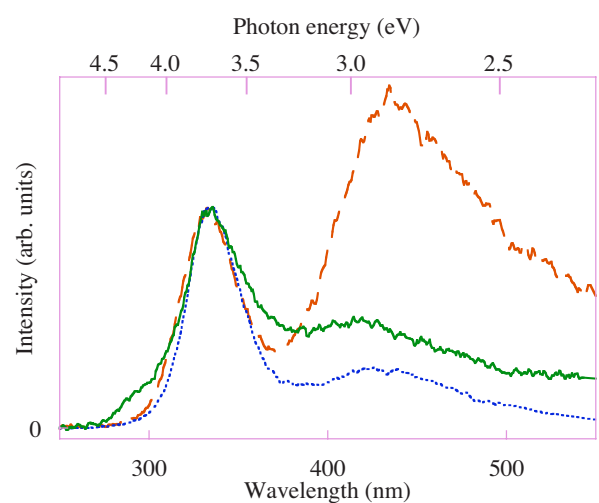

FIG. 4. (Color online) Cathodoluminescence of $\alpha-\mathrm{Al}_{2} \mathrm{O}_{3}$ observed at $1 \mathrm{keV}$ after $21 \mathrm{~h}$ of electron irradiation: sample 2 (dashed line), sample 3 (dotted line), and sample 4 (solid line).

Fig. 7. This variation is in agreement with previous observations. ${ }^{9}$ A supplementary emission is seen at $3.4 \mathrm{eV}$ (Fig. 6). On the curves obtained at $1.9 \mathrm{keV}$, the peak at 3.4 $\mathrm{eV}$ is well resolved; its shape is similar to that of the $\mathrm{F}^{+}$ emission and its intensity increases with the irradiation time [Figs. 6(a) and 6(c)]. In contrast, this peak is not clearly resolved at $1 \mathrm{keV}$ [Figs. 6(b) and 6(d)]. Change in the $\mathrm{F}$ emission shape is also observed; this is a very wide band, spreading on more of $1 \mathrm{eV}$, including nonresolved features situated toward the lower energies. Consequently no width measurement is made for this emission. The intensity ratio $\mathrm{F}^{+} / \mathrm{F}$ varies with the energy of the electrons as seen at room temperature for this sample but it does not vary with the irradiation time.

The optical emissions as a function of the electron current vary according to the value of the incident electron energy. Results obtained for sample 4 at low temperature $(90 \mathrm{~K})$ are plotted in Fig. 8. For 1 and $4 \mathrm{keV}$ incident electrons, the ratio $\mathrm{F}^{+} / \mathrm{F}$ is proportional to the current while for electrons of 1.9 $\mathrm{keV}, \mathrm{F}^{+} / \mathrm{F}$ is inversely proportional to the current. Moreover, the shape of the spectra is different. At $1.9 \mathrm{keV}$, the peak at $3.4 \mathrm{eV}$ is clearly resolved and no structure is seen toward the higher energies of $\mathrm{F}^{+}$emission. The contrary is observed at 1 and $4 \mathrm{keV}$.

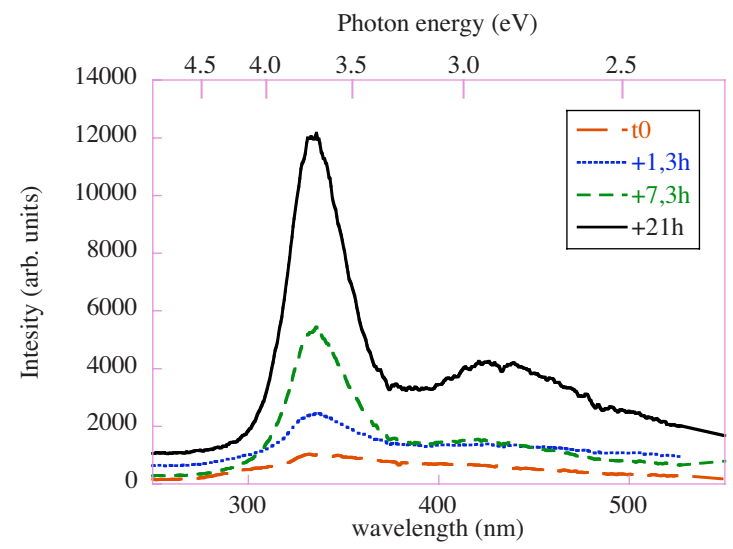

FIG. 5. (Color online) Cathodoluminescence of $\alpha-\mathrm{Al}_{2} \mathrm{O}_{3}$ (sample 3) observed at $1 \mathrm{keV}$, at room temperature, as a function of the irradiation time. 

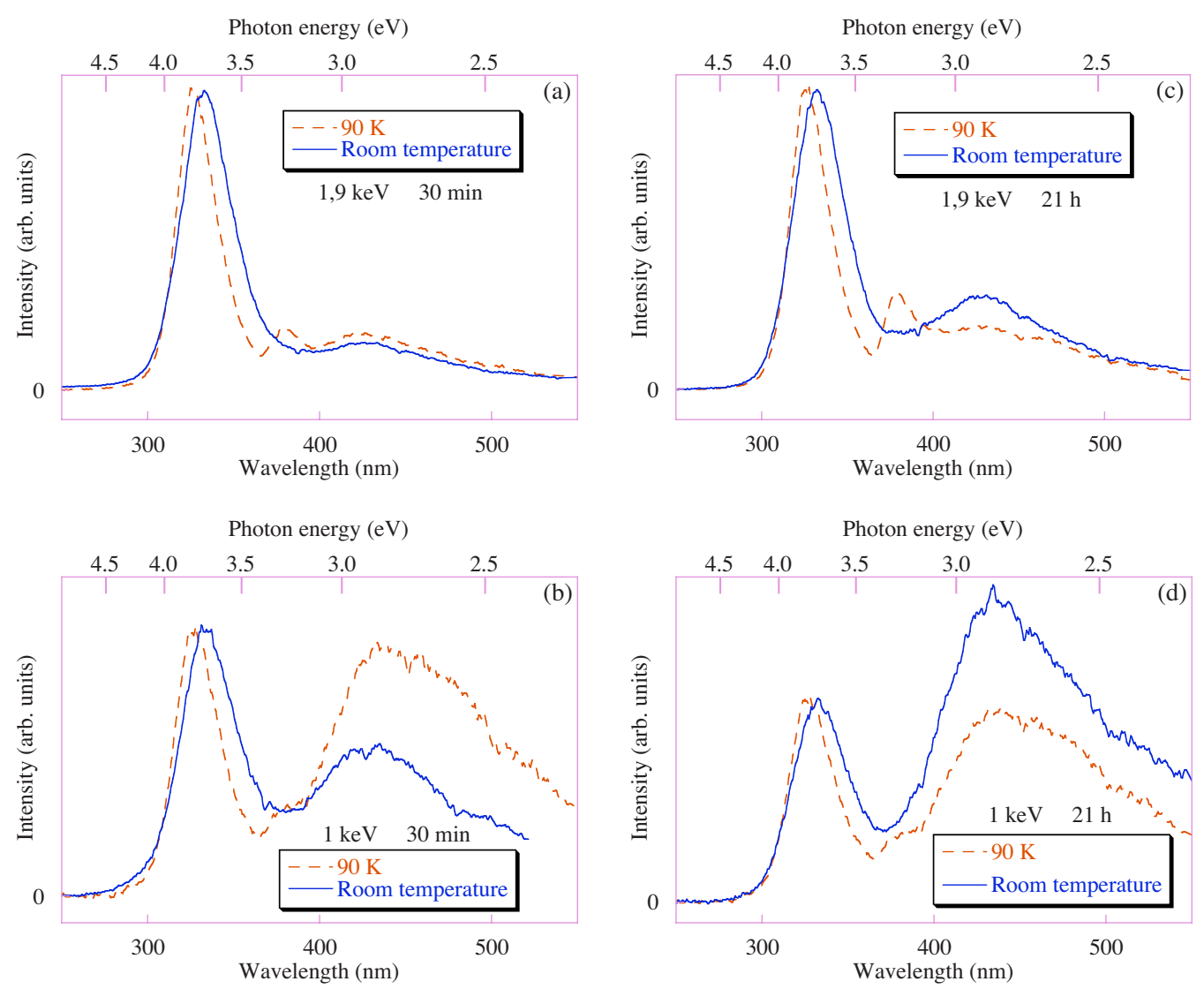

FIG. 6. (Color online) Cathodoluminescence of $\alpha$ - $\mathrm{Al}_{2} \mathrm{O}_{3}$ (sample 2) at room temperature (solid line) and $90 \mathrm{~K}$ (dashed line), at two incident electron energies and two irradiation times: after 30 min irradiation (a) $1.9 \mathrm{keV}$, (b) $1 \mathrm{keV}$; after $21 \mathrm{~h} \mathrm{(c)} 1.9 \mathrm{keV}$, (d) $1 \mathrm{keV}$.

All preceding results concern $\alpha-\mathrm{Al}_{2} \mathrm{O}_{3}$ single crystal. In polycrystalline $\gamma-\mathrm{Al}_{2} \mathrm{O}_{3}$, the cations are distributed in nonequivalent sites. A large variety of defects is present, like vacancies, interstitials, association of point defects. A wide band is observed between 3.8 and $2 \mathrm{eV}$ (Fig. 9). Nonresolved structures are present and the peak characteristic of the $\mathrm{F}^{+}$ emission is not observed. Contrary to the observations for $\alpha-\mathrm{Al}_{2} \mathrm{O}_{3}$ single crystal, the intensity varies linearly with the irradiation parameters. The behavior of amorphous $\mathrm{Al}_{2} \mathrm{O}_{3}$ is very similar to that of the polycrystal.

\section{DISCUSSION}

A significant result of this paper, based on observation of $\mathrm{x}$-ray charge and excitation curves, is the evidence of the presence of charges in the material investigated. Another $\mathrm{x}$-ray emission result concerns the average number of electrons around oxygen. This number is close to unity. Then one deduces that the more stable defects are the $\mathrm{F}^{+}$centers. This result is in agreement with electron-paramagnetic-resonance measurements. ${ }^{10}$

Most of our observations concern the $\mathrm{F}^{+}$and $\mathrm{F}$ defect centers and their change in the presence of charge trapping and detrapping. The intensity of the $\mathrm{x}$-ray emissions due to transitions between a defect state localized in the band gap and a core hole is small with respect to that of the $\mathrm{x}$-ray valence emission band for $\alpha-\mathrm{Al}_{2} \mathrm{O}_{3}$ because the number of the defect states is small. Moreover these emissions are broadened by the Gaussian statistical distribution of the defects in the material. Their intensity is a function of the number of populated defect states. These states can be occupied before irradiation. They can be filled by direct excitation of a core or valence electron or by shake-up effect from the valence band. Under electron irradiation, the oxygen vacancies and $\mathrm{F}^{+}$centers can also trap electrons. These electrons are either primary electrons or secondary electrons generated by

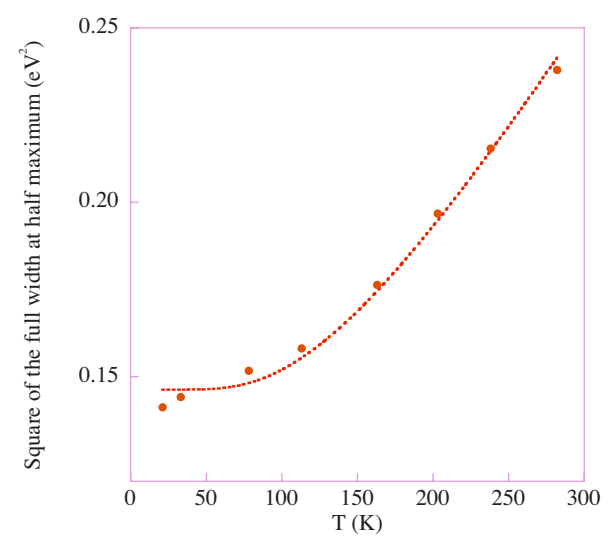

FIG. 7. (Color online) Square of the width of the $3.75 \mathrm{eV}$ cathodoluminescence peak of $\alpha-\mathrm{Al}_{2} \mathrm{O}_{3}$ (sample 3) obtained at 4 $\mathrm{keV}$ and $0.5 \mathrm{~mA}$ as a function of the temperature. The dotted line is a fit with the model described in Ref. 9. 

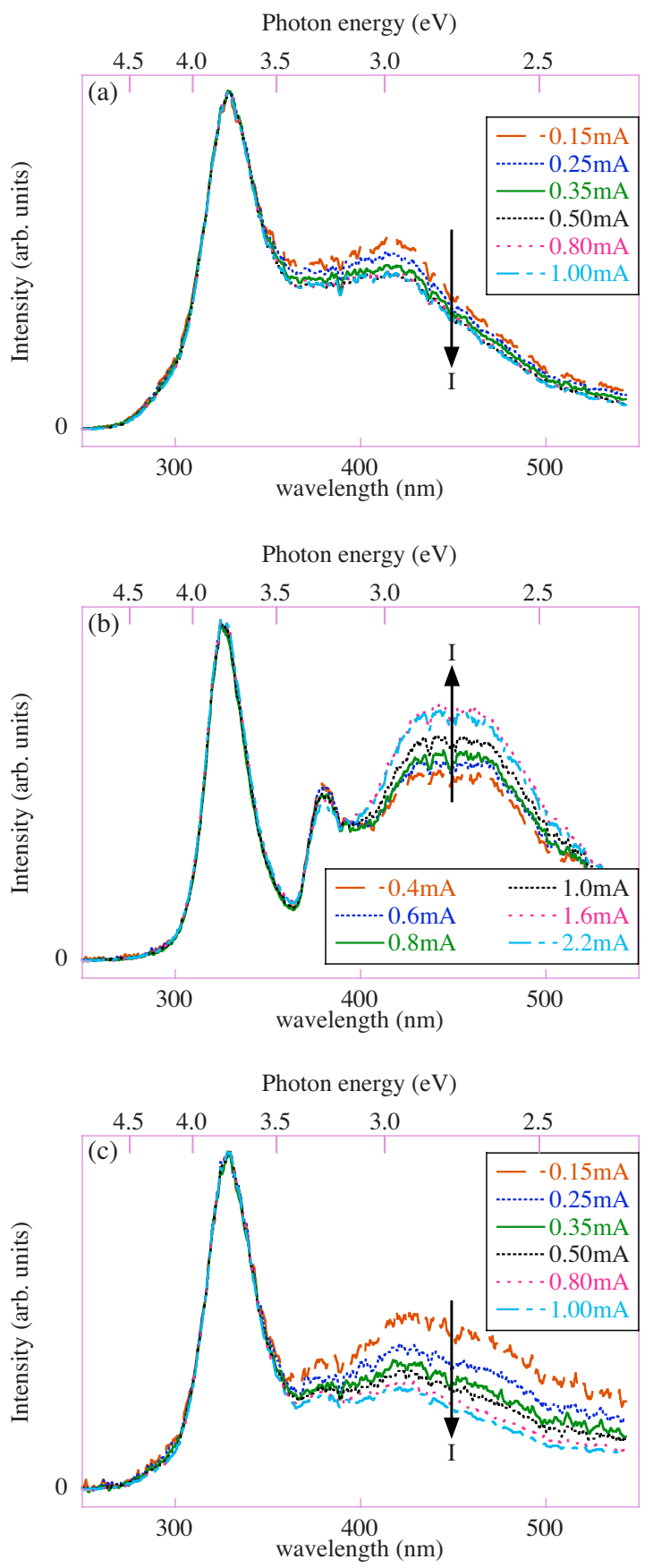

FIG. 8. (Color online) Cathodoluminescence of $\alpha-\mathrm{Al}_{2} \mathrm{O}_{3}$ (sample 4) observed at $90 \mathrm{~K}$ as a function of the electron current: (a) at $4 \mathrm{keV}$; (b) at $1.9 \mathrm{keV}$; and (c) at $1 \mathrm{keV}$ electron energy.

interaction of the primary electrons with the material that had been thermalized in the conduction band.

Among the filling processes of a defect state, only the trapping depends on the temperature and on the number of thermalized electrons, thus on the number of electrons of low energy crossing the material. The trapping depends also on several cross sections: the probability of electron capture from the continuum states to the highly excited levels of the defect state, the probability of the reverse process and the probabilities of radiative and nonradiative de-excitation to the ground state. The intensity of the defect peaks with re-

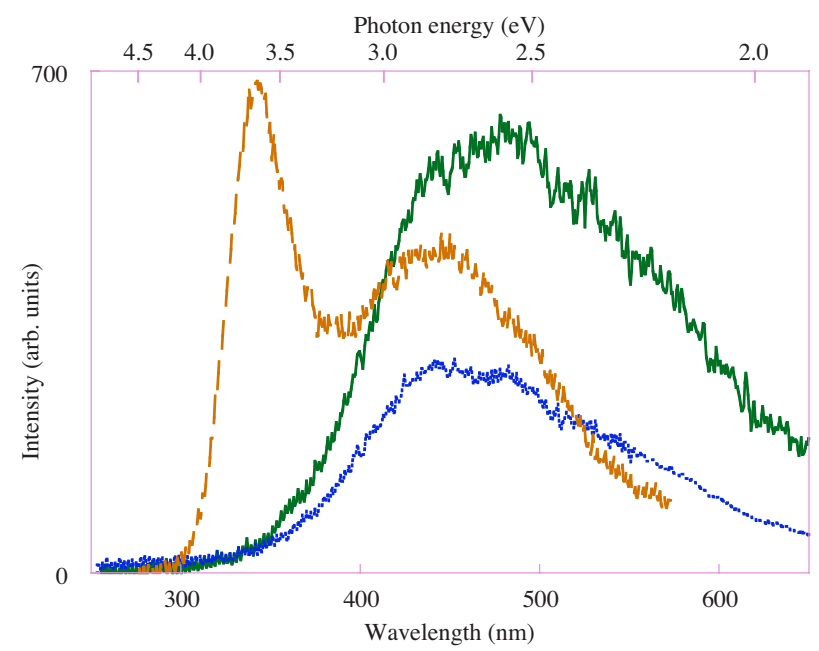

FIG. 9. (Color online) Cathodoluminescence under $3 \mathrm{keV}$ and $0.1 \mathrm{~mA}$ electron irradiation of $\alpha-\mathrm{Al}_{2} \mathrm{O}_{3}$ (sample 2, dashed line), a- $\mathrm{Al}_{2} \mathrm{O}_{3}$ (dotted line), and $\gamma-\mathrm{Al}_{2} \mathrm{O}_{3}$ (solid line).

spect to the intensity of the valence band is observed to increase when the temperature and incident electron energy decrease. The variation with the temperature reveals the trapping of thermalized electrons by the defect.

For the optical spectra, the emission corresponding to the transition between excited and ground levels of $\mathrm{F}^{+}$centers is at $3.75 \mathrm{eV}$. From the Gauss shape of this emission we deduce that the ground and excited levels of $\mathrm{F}^{+}$center are discrete levels not interacting with the continuum states. At low temperature, the $\mathrm{F}^{+}$emission is well resolved and quasisymmetrical because the Gaussian broadening of the emission decreases with the temperature.

The structure at $3.4 \mathrm{eV}$ is observed only at low temperature (Fig. 6). Its intensity changes with the incident electron energy and increases with the irradiation time. It is well resolved at $1.9 \mathrm{keV}$, its shape and width being thus analogous to that of the $\mathrm{F}^{+}$emission. We interpret this structure as due to an aggregate defect, the $\mathrm{F}_{2}^{+}$centers. Indeed, $\mathrm{F}_{2}, \mathrm{~F}_{2}^{+}$, and $\mathrm{F}_{2}^{2+}$ centers, corresponding to two to four electrons trapped at a next-nearest-neighbor anion vacancy pair, were shown to exist. $^{11}$

The structure seen toward the higher energy of the $\mathrm{F}^{+}$ emission at $4.3 \mathrm{eV}$ could be due to an $\mathrm{F}_{2}^{2+}$ center. This peak is observed only for the sample 4 . It is clearly seen at room temperature (Fig. 4). At lower temperatures the shift of the $\mathrm{F}^{+}$emission toward higher energies makes its observation difficult. However, it is still observed at 1 and $4 \mathrm{keV}$ (Fig. 7). In contrast, at $1.9 \mathrm{keV}$, only $\mathrm{F}_{2}^{+}$is present.

The emission of $\mathrm{F}$ centers is strongly broadened at low temperature. Its shape at $50 \mathrm{~K}$ suggests that an unresolved emission is present toward the lower energies. This emission could be due to $F_{2}$ aggregate centers. Because the intensity of emissions associated with the aggregates increases clearly at low temperature, these defects could not be initially present in the sample but be formed under irradiation at low temperature. Indeed, it is known that in the presence of a field, the defects migrate in order to form aggregates.

The relative intensities of the peaks vary at low temperature as a function of incident electron energy (Fig. 8 for 
sample 4). At 1 and $4 \mathrm{keV}$, the $\mathrm{F}_{2}^{2+}$ emission is observed, the $\mathrm{F}_{2}^{+}$emission is weak and the $\mathrm{F}^{+} / \mathrm{F}$ ratio is large. At $1.9 \mathrm{keV}$, the reverse behavior is observed: the structure $\mathrm{F}_{2}^{+}$becomes relatively intense while the $\mathrm{F}_{2}^{2+}$ emission and the $\mathrm{F}^{+} / \mathrm{F}$ ratio decrease. The changes seen at $1.9 \mathrm{keV}$ are characteristic of charge trapping. Consequently, it appears that in our experimental conditions the trapping varies with the electron incident energy in the range between 1 and $4 \mathrm{keV}$.

The dependence of the $\mathrm{F}^{+} / \mathrm{F}$ ratio on electron current densities varying between 0.1 and $2 \mathrm{~mA} / \mathrm{cm}^{2}$ remains weak. However, by following the absolute intensities of the $\mathrm{F}^{+}$and $\mathrm{F}$ emissions, corrected to take into account the background intensity, both emissions increase by following a similar variation at $1.9 \mathrm{keV}$ while the intensity of $\mathrm{F}^{+}$emission increases more rapidly than that of $\mathrm{F}$ emission at 1 and $4 \mathrm{keV}$.

The variations observed with the incident electron energy can be explained as follows: under electron irradiation, emission of secondary electrons takes place from a surface layer whose thickness is equal to the secondary electron escape depth. This depth is known to be in the order of some tens of nanometers for insulators. ${ }^{12}$ In principle, in this layer the sample is positively charged and the secondary emission yield is higher than one. If the primary electrons penetrate deeper in the sample, they charge negatively deeper layers of the material. For a specific amount of incident energy the secondary emission yield tends to unity. Our results suggest that for incident energy around $2 \mathrm{keV}$, the secondary emission yield rapidly decreases and presents a minimum, making the negative charge increases. An analogous behavior has been observed for $\mathrm{MgO}$, irradiated by electrons of energy between 1.1 and $5 \mathrm{keV}$ and current density between 0.02 and $1 \mathrm{~mA} / \mathrm{cm}^{2} .{ }^{13}$ It has been explained by taking into account the elastic diffusion between primary and secondary electrons. This process should induce a spatial dispersion of the secondary electrons and their accumulation in the material. The effect should be observable only with primary electrons of sufficient current density and of incident energy such that their penetration depth is equal to or slightly higher than the escape depth of the secondary electrons.

Due to the absence of the ionic core in the $\mathrm{F}$ and $\mathrm{F}^{+}$ centers, the electrons exert forces on the neighboring ions and the coupling between the electrons present in the $\mathrm{F}$ or $\mathrm{F}^{+}$ centers and the lattice modes is strong. The presence of multiphonon processes between the different defect states is associated with this strong coupling. The strength of the electron-phonon coupling can be estimated from the energy difference between the absorption and the emission, labeled Stokes shift, or energy relaxation $E_{\mathrm{r}}$. Indeed, the energy $E_{\mathrm{r}}$ is related to the Huang-Rhys factor $S_{\mathrm{HR}}$ by $E_{\mathrm{r}}=S_{\mathrm{HR}} / \hbar \omega$, where $\hbar \omega$ is the phonon energy. ${ }^{4}$ The excited levels of $\mathrm{F}^{+}$center contributing to the emission are discrete levels not interacting with the continuum states. In the determination of the Stokes shift, only the absorption to the excited levels must be taken into account. At $77 \mathrm{~K}$ a small increase in the absorption is observed from about $4.2 \mathrm{eV}$, followed by two large maxima at 4.8 and $5.4 \mathrm{eV}$, corresponding to transitions to continuum levels. ${ }^{14}$ The Stokes shift is not precisely known but can be estimated to some tenths of electron volt. The value of the Huang-Rhys factor determines the strength of electron-phonon coupling, i.e., the strength of the coupling between the $\mathrm{F}^{+}$centers and the lattice. For the $\mathrm{F}$ centers, the Stokes shift should be larger, making these defect states more strongly coupled to lattice vibration modes. The Gaussian broadening of the emission bands with the temperature that dominates the other broadening shows also the importance of the electron-phonon interactions.

As already underlined, an $\mathrm{F}$ center results from the trapping of a thermalized electron by an $\mathrm{F}^{+}$center. But an electron of an F center can be detrapped with large probability because the excited levels of $\mathrm{F}$ can interact with the continuum, making possible an electron transfer to the conduction band. Consequently, the variation in the $\mathrm{F}^{+} / \mathrm{F}$ ratio, i.e., the intensity ratio of the 3.75 and $3 \mathrm{eV}$ peaks, directly reveals whether trapping or detrapping are present. A strong change in the $\mathrm{F}^{+} / \mathrm{F}$ ratio is observed at low incident electron energy after a long irradiation but only for sample 2. A very strong variation in the $\mathrm{F}^{+} / \mathrm{F}$ ratio is also observed between the crystal and noncrystallized samples.

Atomic structure effects are governed by the distribution of defects. In the polycrystal and the amorphous, a quasicontinuum of defect states replaces the localized discrete defect states characteristic of crystalline $\alpha-\mathrm{Al}_{2} \mathrm{O}_{3}$. The trapping takes place in this quasicontinuum of states, situated in the upper part of the gap. This quasicontinuum increases strongly the emission width and facilitates the transitions between the levels of the F centers and the conduction band. Consequently, the charge effects are more important in the disordered systems than in single crystals and for sample 1 because it was not annealed. Indeed oscillations of the X-ray emission have been observed at low temperature for this sample. Charge effects are also more important for sample 2 than for samples 3 and 4 because the number of oxygen vacancies is larger for a lower annealing temperature.

In summary, the intensity of the $\mathrm{Al}_{2} \mathrm{O}_{3} \mathrm{~F}^{+}$and $\mathrm{F}$ optical emissions vary widely with the temperature, irradiation conditions, and the sample structure. A decrease in the $\mathrm{F}^{+} / \mathrm{F}$ intensity ratio reveals an increase in the trapping. The density of trapped electrons increases with a decrease in the temperature, a decrease of the incident electron energy and density and and also with an increase in irradiation time and the crystalline disorder. In the limit of incident energies and current densities used in our experiments, the number of trapped electrons is limited and the system remains in an equilibrium state. $^{15}$

\section{CONCLUSION}

X-ray and optical emissions provide direct evidence of the presence of charges in insulators and display the dependence of the charge effect on the various experimental parameters. These emissions make possible the characterization of the defect states. The dynamics of electron trapping and detrapping, that take place between localized defect states and continuum states of insulator oxides, can be deduced from the relative intensities of the $\mathrm{F}^{+}$and $\mathrm{F}$ emissions.

Defects and trapped charges induce a local electric field and a fast electron beam creates a space-charge field. Consequently, phonon-assisted tunneling from a bound state into a free state can occur, favoring the ionization processes or de- 
trapping. On the other hand, because the electron-phonon coupling is strong for the defect levels, multiphonon transitions are present. These transitions compete with the luminescence and increase with the temperature. Their presence is revealed by the broadening and the shape change observed for the optical emissions with an increase in the temperature.
In our experimental conditions, the regime existing between trapping and detrapping induced by electron collisions, multiphonon transitions, and photoconversion leads to an equilibrium under irradiation. Stability conditions can be derived from these experiments.
*Corresponding author; christiane.Bonnelle@upmc.fr

${ }^{1}$ J. H. Harding, Rep. Prog. Phys. 53, 1403 (1990).

${ }^{2}$ P. Jonnard, F. Vergand, C. Bonnelle, E. Orgaz, and M. Gupta, Phys. Rev. B 57, 12111 (1998).

${ }^{3}$ P. Jonnard, C. Bonnelle, G. Glaise, G. Remond, and C. RoquesCarmes, J. Appl. Phys. 88, 6413 (2000).

${ }^{4}$ C. Bonnelle, Phys. Rev. B 81, 054307 (2010).

${ }^{5}$ M. Kefi, P. Jonnard, F. Vergand, C. Bonnelle, and E. Gillet, J. Phys.: Condens. Matter 5, 8629 (1993).

${ }^{6}$ C. Bonnelle, F. Vergand, P. Jonnard, J. M. André, P. F. Staub, P. Avila, P. Chargelègue, M. F. Fontaine, D. Laporte, P. Paquier, A. Ringuenet, and B. Rodriguez, Rev. Sci. Instrum. 65, 3466 (1994).

${ }^{7}$ P. Jonnard, P. Chargelègue, C. Hornbourger, J. Thirion, and F. Vergand, Rev. Sci. Instrum. 67, 2417 (1996).

${ }^{8}$ C. Bonnelle, Microsc. Microanal. 10, 691 (2004).
${ }^{9}$ J. D. Brewer, B. T. Jeffries, and G. P. Summers, Phys. Rev. B 22, 4900 (1980).

${ }^{10}$ R. C. DuVarney, A. K. Garrison, J. R. Niklas, and J. M. Spaeth, Phys. Rev. B 24, 3693 (1981)

${ }^{11}$ B. D. Evans, G. J. Pogatshnik, and Y. Chen, Nucl. Instrum. Methods Phys. Res. B 91, 258 (1994).

${ }^{12}$ NIST electron effective-attenuation-length database, http:// www.nist.gov/srd/nist82.htm

${ }^{13}$ A. Boughariou, F. Hachicha, A. Kallel, and G. Blaise, Nucl. Instrum. Methods Phys. Res. B 240, 697 (2005).

${ }^{14} \mathrm{G}$. Molnar, Ph.D. thesis, Université de Nice-Sophia-Antipolis, 2000.

${ }^{15}$ C. Bonnelle, G. Blaise, C. Le Gresssus, and D. Tréheux, Les Isolants: Physique de la localisation des porteurs de charge. Applications aux phénomènes d'endommagement (Lavoisier, Paris, in press). 